\title{
Experiential Evaluation of Outdoor Radio Source Localization Using Spatial Information of Array
}

\author{
Hiroyuki Tsuji ${ }^{\dagger}$, Panarat Cherntanomwong ${ }^{\ddagger}$, Jun-ichi Takada \\ †New Generation Wireless Communications Research Center \\ National Institute of Information and Communications Technology \\ Yokosuka, Japan \\ Department of International Development Engineering \\ Tokyo Institute of Technology \\ Tokyo, Japan
}

\begin{abstract}
The fundamental performances of a radio source localization system were evaluated using the spatial information of the received signals by array antenna in outdoor experiments. We used the angle-of-arrival-based and newly proposed methods to estimate the location of a terminal. The proposed method of localization is based on the fingerprinting technique and uses the signal subspaces of the received signal by using an array antenna as the feature quantity. We confirmed that the proposed method successfully estimates the location of a terminal through the outdoor experiments, whereas conventional methods, such as angle-of-arrival-based method, fail to accurately estimate the locations of sources under non line-of-sight conditions.
\end{abstract}

Keywords-radio location estimation; array antenna; signal subspace; location fingerprint; angle of arrival

\section{INTRODUCTION}

Localization techniques that use radio signals have recently attracted international attention because mobile terminal location information is considered to be useful in various scenarios, such as vehicle navigation and ubiquitous computing, in addition to the E911 service that detects the precise location of all enhanced 911 (E911) callers that is now required by the U.S. Federal Communications Commission (FCC) [1][2]. Several types of techniques that detect the location of radio sources have already been proposed. The Global Positioning System (GPS) is widely used for detecting positions. However, it does have several restrictions. For example, GPS sometimes suffers from satellite signal shadowing due to buildings and it also needs dedicated devices to establish its location. Systems that allow their location to be performed without the need of GPS at the mobile terminal offer some advantages and have various uses. For this reason, the National Institute of Information and Communications Technology (NICT) is focusing on a localization system that uses an array antenna, because the array antenna can provide several important parameters used for estimating the location of a terminal, such as the angle-of-arrival (AOA) of a received signal at the base station. Therefore, for this paper we evaluated a radio source localization system that uses an array antenna through experiments in which AOA-based and a newly proposed localization methods were applied to estimate the location of a radio source. The proposed localization method in this study is classified as a location fingerprinting technique.
Although there are many studies that use the fingerprinting technique, they do not always effectively take advantage of the information of the received signal. A method is proposed here that uses the information from the signal subspace of a received signal at the array antenna, which includes both the signal strength and the spatial information, and the subspace fitting approach to identify the location of a terminal [3].

We applied both the AOA-based and fingerprinting-based methods to an outdoor mobile localization system and confirmed the effectiveness and validity of the proposed method through outdoor experimentation.

\section{Estimation OF LOCATION OF MOVING CAR}

\section{A. Race Car Positioning System}

The Motor Sports Radio Association (MoSRA) in Japan has been discussing the use of a location display system for race cars to attract more people to motor sports by promoting safe and efficient automobile competitions at racing circuits. The ultimate objective of the system is to obtain all the locations of the race cars in real-time and to display their locations. NICT proposed the race car localization system shown in Fig. 1, in which each race car is equipped with a transmitter and the array antenna estimates the locations of the cars on the circuit. Fixed stations that send beacon signals are also used as reference points. The main feature of the proposed system is that a simple transmitter can reduce the load weight of race cars, and each transmitter does not need to transfer its location information because the system can use the array antenna to estimate the locations of the cars.

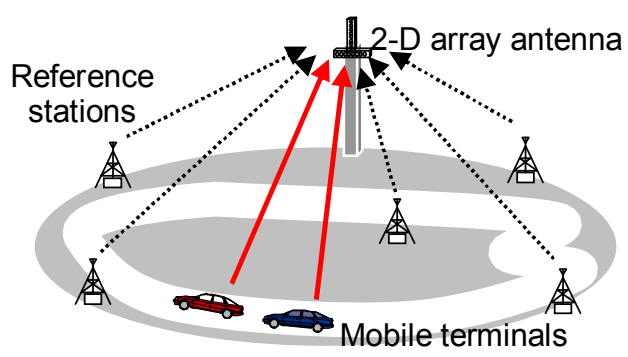

Figure 1. Conceptual diagram of proposed mobile localization system. 


\section{B. Motegi Circuit Localization Experiment}

The MoSRA and NICT conducted an experimental test at the Twin-Ring Motegi Circuit to evaluate the effectiveness of a localization system using an array antenna. Since the main purpose of the experiment was to examine the feasibility and effectiveness of this localization system, the array antenna received and recorded the signals from stationary transmitters (cars) on the circuit from different locations. Then the data were analyzed to obtain their exact locations.

Fig. 2 shows the locations of the transmitters and the array antenna on the circuit. We defined the origin $(0,0,0)$ at the start line of the course and put $\mathrm{X}-\mathrm{Y}-\mathrm{Z}$ coordinates at right angles to the course, along the course, and heightwise, respectively, as shown in Fig. 2. Table I shows the different X-Y-Z positions where the transmitters and the array antenna were located and the horizontal and vertical angles between the array antenna and the transmitters. The locations numbered from -150 to P550 in Fig. 2 were within the line of sight, while those numbered P600 and P700 or after P700 were not.

The receiving array antenna equipment consists of two units of the linear array antenna forming a $T$-shape, one laid horizontally and the other place vertically on top and in the center of the first one forming right angles, as shown in Fig. 3. Its specifications are listed in Table II. The received signals were sampled by A/D converters at $4.375 \mathrm{MHz}$ with 12 bits and stored in the recorder. The array antenna was pointed towards reference station 3 as the zero degrees angle of the AOA. Fig. 4 shows an overview of the experimental field from the receiving point.

The transmitting antenna was put on the roof of the car at a height of $1.5 \mathrm{~m}$ and the cars position was changed according to the locations in Fig. 2. Three fixed reference stations with different frequencies were also placed at the locations indicated as Reference Stations 1-3 in Fig. 2.

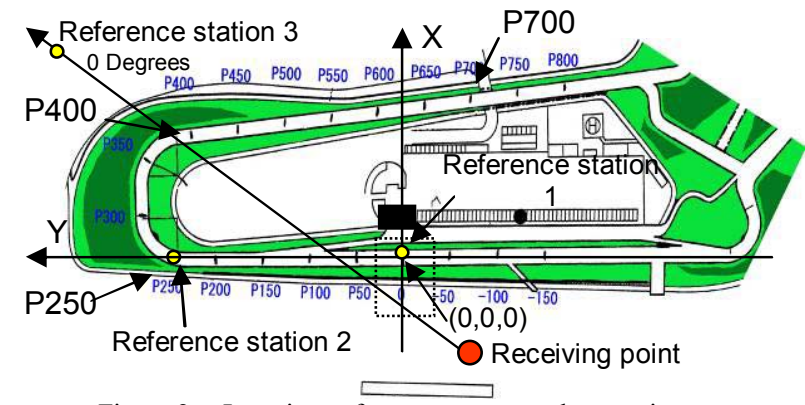

Figure 2. Locations of array antenna and transmitters.

TABLE I. POSITION COORDINATES OF LOCATIONS IN FIG. 2.

\begin{tabular}{|l|l|l|l|l|l|}
\hline \multirow{2}{*}{ Locations } & \multicolumn{2}{|c|}{ X-Y-Z coordinate } & \multicolumn{2}{c|}{ Angles } \\
\cline { 2 - 6 } & $\boldsymbol{x}[\mathbf{m}]$ & $\boldsymbol{y}[\mathbf{m}]$ & $\boldsymbol{z}[\mathbf{m}]$ & H $[\mathbf{d e g}]$ & $\mathbf{V}[\mathbf{d e g}]$ \\
\hline Array Antenna & -107.0 & -78.0 & 32.1 & - & - \\
\hline Ref. Station 1 & 8.0 & 0.0 & 1.5 & -17.64 & 12.25 \\
\hline Ref. Station 2 & 7.8 & 244.0 & 1.5 & 18.09 & 5.04 \\
\hline Ref. Station 3 & 231.0 & 358.0 & 8.2 & 0.00 & 2.44 \\
\hline -50 & 0.0 & -50.0 & 1.5 & -37.9 & 14.6 \\
\hline P60 & 0.0 & 60.0 & 1.5 & 0.7 & 7.5 \\
\hline P90 & 0.0 & 90.0 & 1.5 & 5.0 & 6.8 \\
\hline P250 & 6.4 & 249.0 & 1.5 & 18.8 & 5.1 \\
\hline P400 & 115.0 & 207.0 & 1.5 & 2.9 & 5.2 \\
\hline P600 & 135.0 & 8.2 & 1.5 & -30.8 & 6.4 \\
\hline P700 & 145.0 & -91.2 & 1.5 & 30 & 6.7 \\
\hline
\end{tabular}

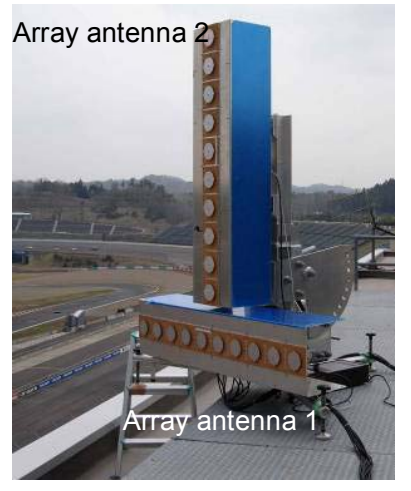

Figure 3. Photograph of receiving array antenna.

TABLE II. RECEIVING ARRAY ANTENNA SPECIFICATIONS

\begin{tabular}{|l|l|}
\hline Center Frequency & $1.74 \mathrm{GHz}$ \\
\hline Receiving antenna & 10 elements linear array antenna \\
\hline Element spacing & 0.8 wavelength of carrier wave \\
\hline Type of antenna element & Patch antenna \\
\hline IF output frequency & $1.9648 \mathrm{MHz}$ \\
\hline
\end{tabular}

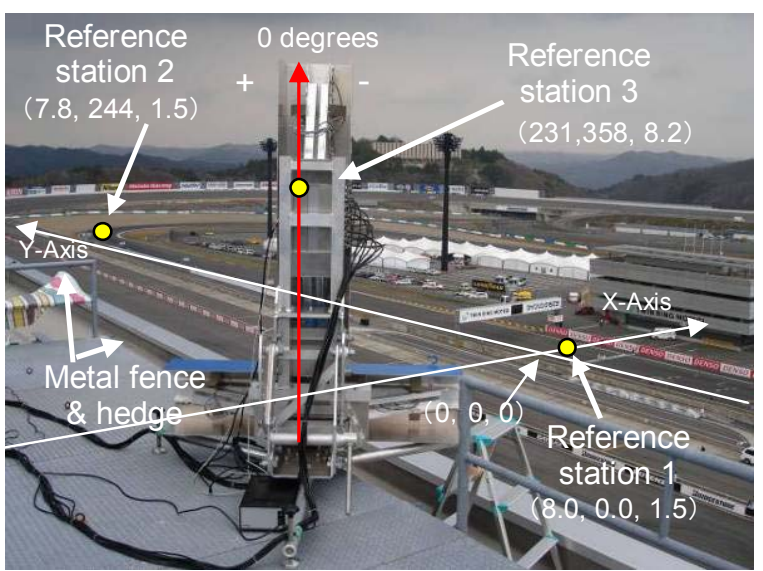

Figure 4. Photograph of experimental field (West course of Twin-Ring Motegi).

\section{LOCATION ESTIMATION USING AOA-BASED TECHNIQUE}

\section{A. Data measurement and AOA estimation methods}

The two units of the array antennas received a total of 19968 snapshots at each transmitting position by changing the location of the transmitter. This snapshot total was divided into 78 blocks with 256 samples, and to each block a fast Fourier transform (FFT) was applied to separate the target signal from the measurement data. We used three AOA estimation algorithms: 1) a beamformer method with a $20 \mathrm{~dB}$ Chebyshev distribution, 2) a multiple signal classification (MUSIC), and 3) MUSIC with spatial smoothing to the data, and obtained the horizontal and vertical AOA estimations of the impinging signals. As for details of the methods, refer to other research papers, such as [4] and [5].

Figs. 5 and 6 show the horizontal and vertical AOA estimation results using the first two methods when the transmitter was located at P90. As shown in these figures, the estimated AOA results from the first method roughly correspond with those from the second one. In addition, the results from the third method also agree with the other results. 

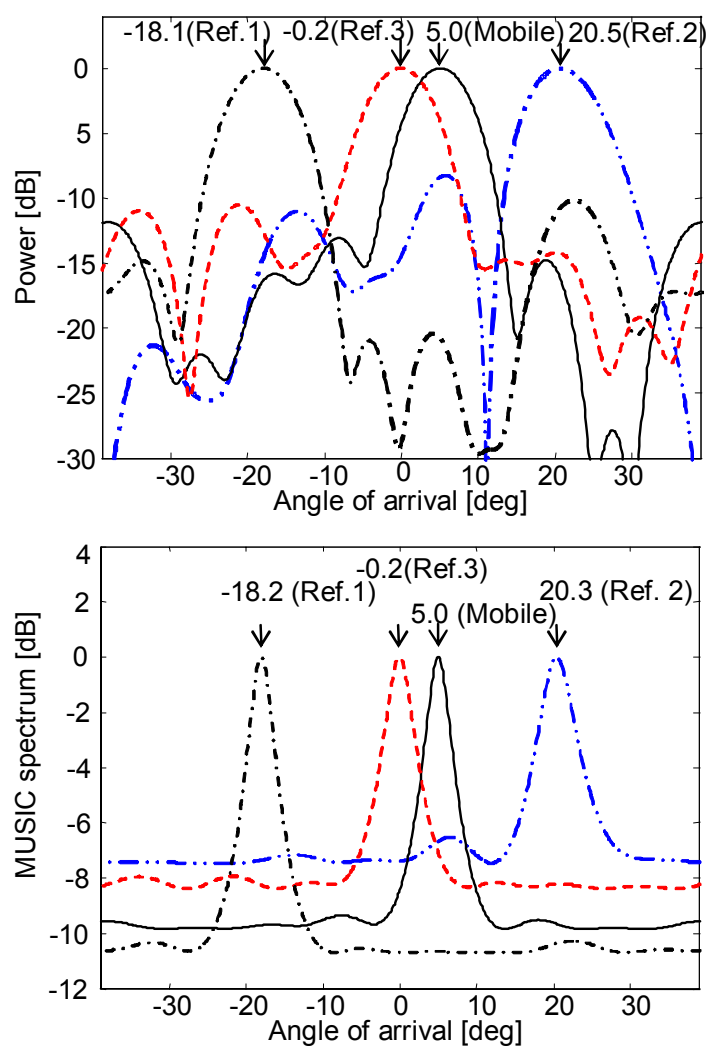

Figure 5. AOA estimation results of horizontal planes by the beamformer and MUSIC methods.
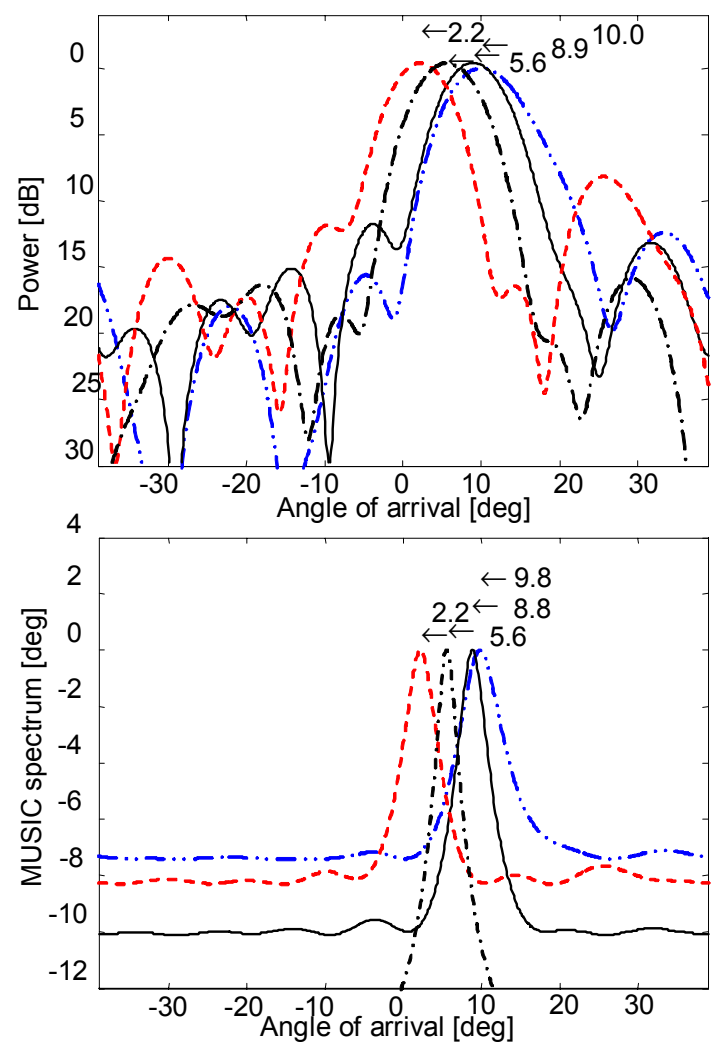

Figure 6. AOA estimation results of vertical planes by the beamformer and MUSIC methods.

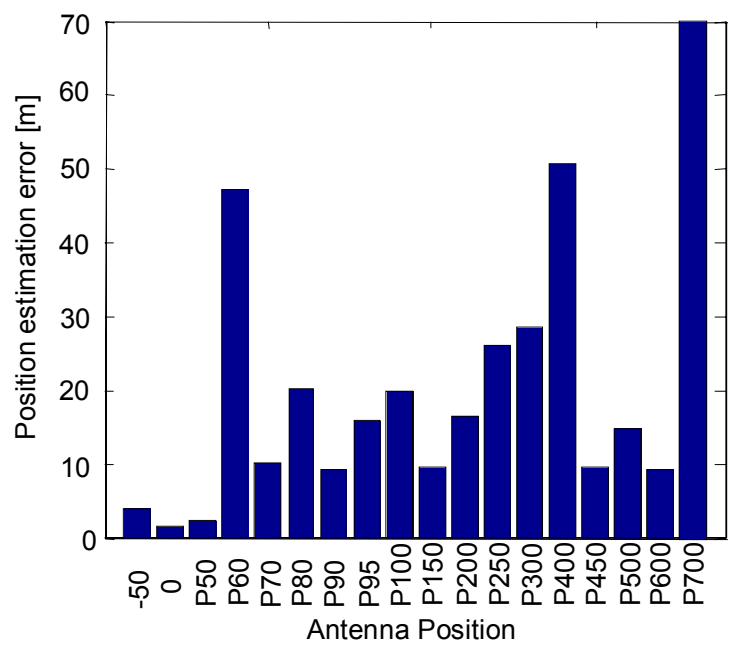

Figure 7. Estimation errors of mobile terminal positions.

\section{B. Location estimation by estimated AOA information}

The location of the transmitter could be geometrically calculated using the AOA estimations obtained by the twodimensional array antenna combining the two linear arrays.

Fig. 7 shows some of the results for the estimated location errors of the transmitter obtained by calculating the geometrical position of the transmitter using the three averaged AOA estimation results of the beamformer method. As you can see from the results, it was confirmed that the average estimation error was roughly within $10 \mathrm{~m}$, except at P60, P400 and P700. This was attributed to the fact that P400 and P700 were not within line of sight and that there were some objects around P60, thereby causing multipath signals.

Let us summarize the results obtained by AOA-based method in the experiment:

- In the experiment, the AOA-based localization technique could estimate the locations of the terminal within an accuracy of about $10 \mathrm{~m}$ under line-of-sight conditions.

- The AOA-based localization technique was not practical for estimating the locations of the terminal when the transmitters were not within the line-of-sight or in a multipath environment.

\section{LOCATION ESTIMATION USING FINGERPRINTING PATTERN MATCHING}

\section{A. Subspace matching for fingerprinting technique}

The basic idea of the fingerprinting approach is to match the patterns based on the positioning technique, which generally uses the received signal strength. Therefore, fingerprinting techniques generally require a database for the matching patterns based on the positioning technique. Our approach uses the principal angles between the signal subspaces of the spatial covariance matrices of the signals received by the array antennas to measure the similarity between the signals transmitted from two locations. We specifically use a subspace matching approach to identify the 
location of a transmitter [3]. The definition for the principal angles for the subspace matching is as follows [6]:

Let $\boldsymbol{E}_{1}$ and $\boldsymbol{E}_{2}$ be the subspaces in the $M$ dimensional complex vector spaces with the dimension $d$. The principal angles $\alpha_{i}$ between $\boldsymbol{E}_{1}$ and $\boldsymbol{E}_{2}$ are defined recursively as follows. For $\|\boldsymbol{u}\|=\|v\|=1$, we define

$$
\begin{gathered}
\cos \alpha_{i}=\min _{u \in E_{1}} \min _{v \in E_{2}}\left|\boldsymbol{u}^{H} \boldsymbol{v}\right|=\left|\boldsymbol{u}_{i}^{H} \boldsymbol{v}_{i}\right|, \quad i=1, \cdots, d \\
\boldsymbol{u}^{H} \boldsymbol{u}_{j}=\boldsymbol{v}^{H} \boldsymbol{v}_{j}=0, \quad j=1, \cdots, i-1
\end{gathered}
$$

Note that the principal angles $\alpha_{1}, \alpha_{2}, \cdots, \alpha_{d}$ have the property $0 \leq \alpha_{1} \leq \cdots \leq \alpha_{d} \leq \pi / 2$, and $\alpha_{d}$ indicates the largest deviation of $\boldsymbol{E}_{1}$ from $\boldsymbol{E}_{2}$.

In our approach, we used the principal angle between the subspaces of the covariance matrix of the array signals as the spatial fingerprint of the transmitter.

\section{B. Location estimation with fingerprinting pattern matching}

We used the same received data for the proposed fingerprinting technique as we did with the AOA-based localization, and calculated the signal subspaces with dimension $d$ from the eigenvalue decomposition of the covariance matrix of the received signals. The covariance matrix was obtained by combining two sets of the observed data from the horizontal and vertical array antennas.

Initially we established a database of the signal subspaces with an index of the transmitting locations. We then sampled the data sent from each transmission point again using the array antenna and calculated the principal angle between the subspace at every location index from the database and that of the received signal. The likeliest location of a transmitter could be identified by finding the minimum value of the principal angle or the maximum value of the cosine of the principal angle between the signal subspaces of the database and the observed signals. The locations estimated from the first result are shown in Fig. 8. The vertical axis indicates the estimated locations and the horizontal axis indicates the location indexes of the database. The dimension of the covariance matrix was 16 and there were eight elements for each array antenna. There were 256 observed data points for estimating the covariance matrix. As shown in Fig. 8, we were able to identify the exact location of a transmitter by finding the minimum principal angle even though P700 was not within the line-of-sight.

Fig. 9 shows the calculated cosine of the principal angles between the subspaces of the database and location P80. The horizontal axis in Fig. 9 represents the location indexes of the database and the vertical axis shows the cosine of the calculated principal angles. Our method could distinguish between the close locations around P80, such as P70 and P90. Another peak was observed at P400 in Fig. 9. That was attributed to the fact that P80 and P400 and the array antenna were almost linearly aligned.

\section{CONCLUSiON}

We propose a method for localization that uses a subspace fitting approach and reported on the fundamental performances of the AOA-based and proposed localization methods from outdoor experiments. The experimental results proved the effectiveness of the proposed method even though the locations of the transmitter were not within the line of sight.

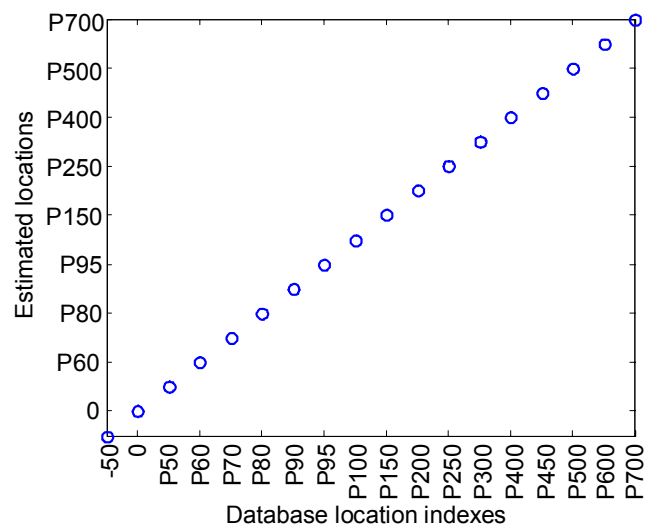

Figure 8. Estimated terminal locations.

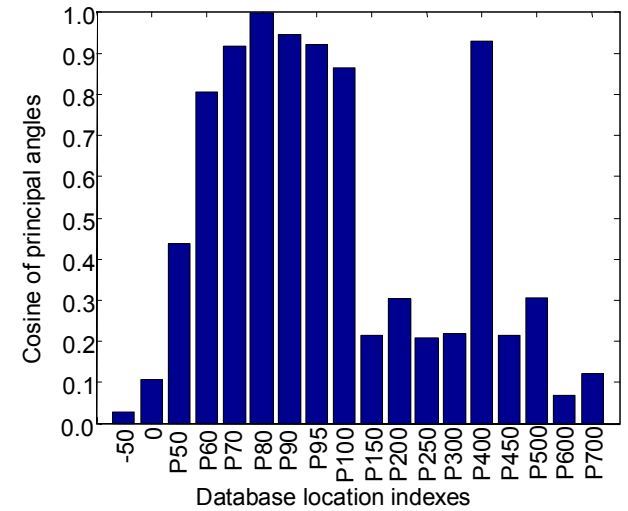

Figure 9. Calculated cosine of principal angles between signal subspaces of database and measured signals (for transmitter located at P80).

\section{REFERENCES}

[1] T. S. Rappaport, J. H. Reed, and B. D. Woerner," "Position location using wireless communications on highways of the future," IEEE Communications Magazine, vol. 34, pp. 30-41, Oct. 1996.

[2] J. H. Reed, K. J. Krizman, B. D. Woerner, and T. S. Rappaport, “An overview of the challenges and progress in meeting the E-911 requirement for location service," IEEE Communications Magazine, vol. 34, pp. 30-37, April 1998.

[3] M. Nezafat, M. Kaveh, and H. Tsuji, "Indoor localization using a spatial channel signature database," IEEE Antennas and Wireless Propagation Letters, vol. 5, Issue 1, pp. 406-409, Dec. 2006.

[4] R. O. Schmidt, "Multiple emitter location and signal parameter estimation," IEEE Trans., Antenna and Propagation, vol. AP-34, no. 3, pp. 276-280, March 1986.

[5] S. U. Pillai and B. H. Kwon, "Forwar-backward spatial smoothing techniques for the coherent signal identification," IEEE Trans. Acoust., Speech, Signal Processing, vol. 37, pp. 8-15, Jan. 1989.

[6] G. Golub and C. V. Loan, Matrix Computations, The Johns Hopkins University Press, 1996. 